\title{
Multivariable Fuzzy Control Based Mobile Robot Odor Source Localization via Semitensor Product
}

\author{
Ping Jiang, ${ }^{1,2}$ Yuzhen Wang, ${ }^{2}$ and Aidong Ge ${ }^{2}$ \\ ${ }^{1}$ School of Electronic and Engineering, University of Jinan, Jinan, Shandong 250022, China \\ ${ }^{2}$ School of Control Science and Engineering, Shandong University, Jinan, Shandong 250061, China
}

Correspondence should be addressed to Ping Jiang; cse_jiangp@ujn.edu.cn

Received 8 April 2015; Accepted 18 June 2015

Academic Editor: Xinguang Zhang

Copyright (C) 2015 Ping Jiang et al. This is an open access article distributed under the Creative Commons Attribution License, which permits unrestricted use, distribution, and reproduction in any medium, provided the original work is properly cited.

In order to take full advantage of the multisensor information, a MIMO fuzzy control system based on semitensor product (STP) is set up for mobile robot odor source localization (OSL). Multisensor information, such as vision, olfaction, laser, wind speed, and direction, is the input of the fuzzy control system and the relative searching strategies, such as random searching (RS), nearest distance-based vision searching (NDVS), and odor source declaration (OSD), are the outputs. Fuzzy control rules with algebraic equations are given according to the multisensor information via STP. Any output can be updated in the proposed fuzzy control system and has no influence on the other searching strategies. The proposed MIMO fuzzy control scheme based on STP can reach the theoretical system of the mobile robot OSL. Experimental results show the efficiency of the proposed method.

\section{Introduction}

In natural world, many organisms such as drosophila, moth, and lobster use olfaction or/and vision cues to find the same species, avoid predators, exchange information, and search for food $[1-3]$. Inspired by those biological activities, in the early 1990s researchers started to build single or multiple mobile robots with onboard odor sensors or/and winds sensor to accomplish the odor source localization (OSL) task. Existing methods can be categorized along two lines. One is olfaction-based method, which mainly uses olfaction or/and wind information to search for gas sources without visual information. The other is vision-based method, which takes the visual information as an assistant of olfaction to accomplish the OSL task. Most work has been focused on the first field and it has become a mature and popular filed. However, the vision-based method is immature and needs to do deep study due to the late beginning. Russell [4], Meng and Li [5], Lilienthal et al. [6], Naeem et al. [7], Kowadlo and Russell [8], and Ishida et al. [9] have given relative reviews about mobile robot OSL from a different angle or application. The interested reader is referred to [4-9] for a comprehensive review of olfaction-based mobile robot OSL. Compared with organisms, robots can be deployed quickly, maintained at low cost, and work for a long time without fatigue. Moreover, they can enter the dangerous or harmful areas. Mobile robot OSL is a multidisciplinary research field with wide potential applications, such as judging toxic/harmful gas leakage location, checking contraband (e.g., heroin), locating unexploded mines and bombs, and fighting against terrorist attacks.

It is well known that human beings normally first look around to search for the most potential region or object and then identify whether the region or object is an odor source by olfaction. Vision contains abundant information, so visual sensor could be a good assistant of olfaction for mobile robot OSL. Meanwhile, large amount of leakage accidents indicate that some devices are more likely to leak, such as valves, bottles, and pipelines. In this paper, such devices are called potential gas sources and the areas which contain such devices are called plausible areas. It would improve the searching efficiency if these potential gas sources are recognized accurately and the plausible areas are determined rapidly in the searching process.

In recent years, a few researchers attempted to integrate vision and olfaction to localize the odor source. Kowadlo et al. [10] took crackles as the vision feature assisting olfaction to search for the odor source. Ishida et al. [11] proposed a color-based algorithm to deal with the vision information in 
the searching process. These methods were verified in the experiments, which indicate that vision as an assistant of olfaction for mobile robot OSL is efficient. Inspired by these researches, Jiang et al. [12] proposed a support vector machine based algorithm to localize an odor source and the author also presented a top-down visual attention mechanism-based algorithm [13] for mobile robot OSL. And then least square estimation was used to fuse the vision and olfaction information to accomplish the OSL task in stable airflow environment [14]. Meanwhile, Jiang and Zhang [15] attempted to integrate the vision and olfaction using subsumption architecture to accomplish the OSL task. However, how to fuse the uncertainty, ambiguity, vagueness, incompleteness, and granularity of the multisensor information from the mobile robot, especially vision and olfaction information, needs further study from the deep analysis to those few reports. It is noteworthy that multisensor data fusion is developed in recent years and new fusion algorithms and models are constantly emerging such as Dempster-Shafer evidence theory, probability theory, fuzzy theory, possibility theory, rough set theory, and the improved algorithms of these methods [16, 17]. Meanwhile, these methods have been successfully used in many fields, such as image processing, fault diagnosis, and target tracking. Inspired by these successful cases, we attempt to set up a multivariable fuzzy control system based on semitensor product for mobile robot OSL by fusing multisensor information and obtain some interesting results.

Fuzzy control as an intelligent control strategy needs no precise mathematical model for the objective system. They have found a great variety of applications ranging from control engineering, qualitative modeling, pattern recognition, signal processing, machine intelligence, and so on [18, 19]. In particular, fuzzy logic control (FLC), as one of the earliest applications of fuzzy sets and systems, has become one of the most successful applications. In fact, FLC has been proved to be a successful control approach to many complex nonlinear systems or even nonanalytic ones. The fuzzy control algorithm consists of a set of heuristic control rules, and fuzzy sets and fuzzy logic are used, respectively, to represent linguistic terms and to evaluate the rules. Since then, fuzzy logic control has attracted great attention from both academic and industrial communities and a lot of excellent books and tutorial articles on the topic have been published. However, it is difficult to infer the proper control input for a multivariable system since the dimension of its relation matrix is very large. The high dimensionality of the relation matrix might lead to not only computational difficulties but also memory overload. To solve this problem, a fuzzy control algorithm by which the multivariable fuzzy system is decomposed into a set of one-dimensional systems $[18,19]$. The decomposition of control rules is preferable since it alleviates the complexity of the problem.

Recently, the semitensor product (STP) of matrices was proposed in [20]. And up to now, it has been widely applied in many fields, such as boolean network [21, 22] and coloring problems [23]. The logic expression can be expressed into an algebraic form by constructing its structure matrix. In [22], the observed data was expressed into a twovalued algebraic form. For the mobile robot odor source localization different sizes of the multisensor information play the different roles in the searching process. Therefore, the multisensor information for the mobile robot odor source localization cannot be divided into two-valued true and false cases simply. This multisensor information is expressed as multivalued algebraic form according to the actual demand. It is noted that the fuzzy logic also can be considered as an extended mix-valued logic in which the truth-values are the ones of memberships of all the elements in a fuzzy set, and the complex reasoning process can be converted into a problem of solving a set of algebraic equations via STP, which greatly simplifies the process of logical reasoning.

In this paper, we attempt to set up a multi-input multioutput (MIMO) fuzzy control framework based on STP for the mobile robot OSL. The multisensor information obtained by the mobile robot is the inputs and the relative searching strategies are the outputs. Several interesting results are obtained. The main contributions of this paper are as follows:

(1) A MIMO fuzzy control system is set up for the mobile robot OSL.

(2) Fuzzy control rules with algebraic equations are given according to the multisensor information.

(3) Any output can be updated in this framework and has no influence to the others.

(4) The proposed method based on MIMO fuzzy control scheme via STP for mobile robot OSL can reach the theory of this field.

The rest of this paper is organized as follows. Section 2 provides some necessary preliminaries on the semitensor product of matrices and the expression of logical function and logical variables. Section 3 presents the proposed algorithms for mobile robot OSL. Section 4 shows experimental results and analysis and the conclusion is given in Section 5 .

\section{Matrices with Logical Variables}

First, some notations are introduced, which will be used in this paper:

(i) $\delta_{k}^{i}$ : the $i$ th column of the identity matrix $I_{k}$.

(ii) $\Delta_{k}:=\left\{\delta_{k}^{i} \mid i=1,2, \ldots, k\right\}$; especially, $\Delta:=\Delta_{2}$.

(iii) $\mathscr{D}:=\{1,0\}$; to use matrix expression, " 1 " and " 0 " can be expressed with the following vectors, respectively: $1 \sim \delta_{2}^{1}, 0 \sim \delta_{2}^{2}$.

(iv) $\mathscr{D}_{k}:=\{1,(k-2) /(k-1),(k-3) /(k-1), \ldots, 0\}, k \geq 2$.

(v) A matrix $L \in \mathbb{R}^{m \times n}$ is called a logical matrix if the columns of $L$, denoted by $\operatorname{Col}(L)$, are of the form $\delta_{n}^{k}$; that is, $\operatorname{Col}(L) \subset \Delta_{n}$.

(vi) Let $\mathscr{L}_{n \times r}$ denote the set of $n \times r$ logical matrices; if $L \in \mathscr{L}_{n \times r}$, by definition, it can be expressed as $L=\left[\begin{array}{llll}\delta_{n}^{i_{1}} & \delta_{n}^{i_{2}} & \cdots & \delta_{n}^{i_{r}}\end{array}\right]$; for the sake of compactness, it is briefly denoted as $L=\delta_{n}\left[\begin{array}{llll}i_{1} & i_{2} & \cdots & i_{r}\end{array}\right]$.

(vii) Each $k$-valued logical value with a vector can be denoted as $(k-i) /(k-1) \sim \delta_{k}^{i}, i=1,2, \ldots, k$; then, $\mathscr{D}_{k} \sim \Delta_{k}$. 


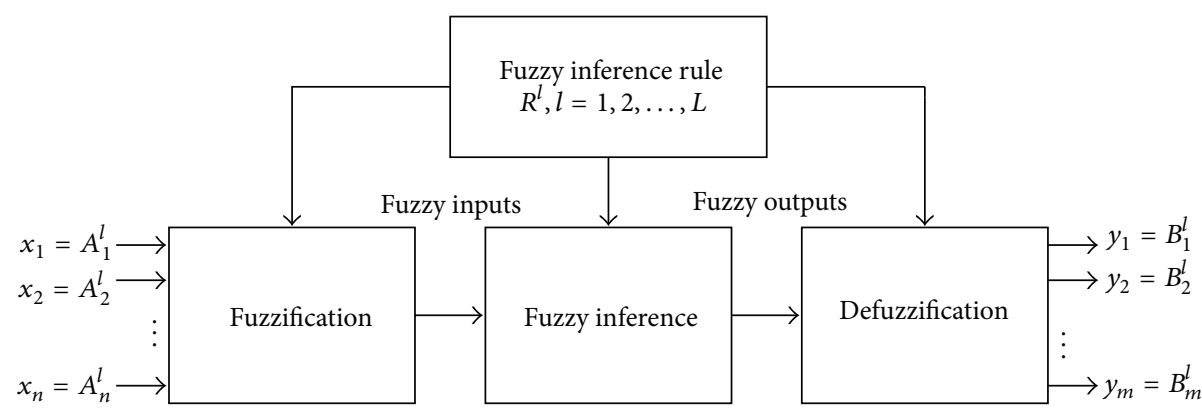

Figure 1: The fuzzy control scheme.

In the following, we recall some definitions and basic properties about the STP [20].

Definition 1. Let $A \in \mathbb{R}^{m \times n}$ and $B \in \mathbb{R}^{p \times q}$. Let $s=\operatorname{lcm}(n, p)$ denote the least common multiple of $n$ and $p$. Then, the semitensor product of $A$ and $B$ is defined as

$$
A \ltimes B=\left(A \otimes I_{s / n}\right)\left(B \otimes I_{s / p}\right),
$$

where “ $\otimes$ " is the Kronecker product.

Remark 2. It is noted that when $n=p$, the STP of $A$ and $B$ becomes the conventional matrix product. Hence, the STP is a generalization of the conventional matrix product. Because of this, we can omit the sign " $\square$ " without confusion.

Definition 3. A swap matrix $W_{[m, n]}$ is an $m n \times m n$ matrix. Its rows and columns are labeled by double index $(i, j)$, the columns are arranged by the ordered multi-index $\operatorname{Id}(i, j ; m, n)$, and the rows are arranged by the ordered multi-index $\operatorname{Id}(J, I ; n, m)$. Then the elements at position $[(I, J),(i, j)]$ are

$$
w_{(I, J)(i, j)}=\delta_{i, j}^{I, J}= \begin{cases}1, & I=i, J=j, \\ 0, & \text { others. }\end{cases}
$$

Remark 4. Let $X \in \mathbb{R}^{n}$ and $Y \in \mathbb{R}^{m}$ be column vectors; then $W_{[n, m]} X Y=Y X$. Let $X_{i} \in \mathbb{R}^{n_{i}}, i=1,2, \ldots, k$, be column vectors; then $\left[I_{n_{1} n_{2} \cdots n_{t-1}} \otimes W_{\left[n_{t}, n_{t+1}\right]}\right] X_{1} \cdots X_{t-1} X_{t} X_{t+1} \cdots X_{k}=$ $X_{1} \cdots X_{t-1} X_{t+1} X_{t} \cdots X_{k}$.

Let $x_{i} \in \mathscr{D}_{k_{i}}, i=1, \ldots, n$ and $y_{j} \in \mathscr{D}_{s_{j}}, j=1, \ldots, m$. Assume that a logic mapping,

$$
F: \mathscr{D}_{k_{1}} \times \cdots \times \mathscr{D}_{k_{n}} \longrightarrow \mathscr{D}_{s_{1}} \times \cdots \times \mathscr{D}_{s_{m}} \text {, }
$$

can be expressed as

$$
\begin{gathered}
y_{1}=f_{1}\left(x_{1}, x_{2}, \ldots, x_{n}\right), \\
y_{2}=f_{2}\left(x_{1}, x_{2}, \ldots, x_{n}\right), \\
\vdots \\
y_{m}=f_{m}\left(x_{1}, x_{2}, \ldots, x_{n}\right),
\end{gathered}
$$

where $f_{j}: \mathscr{D}_{k_{1}} \times \cdots \times \mathscr{D}_{k_{n}} \rightarrow \mathscr{D}_{s_{j}}, j=1, \ldots, m$.
Lemma 5. Any logical function $y=F\left(x_{1}, x_{2}, \ldots, x_{n}\right)$ can be uniquely expressed into the multilinear form of

$$
y=F\left(x_{1}, x_{2}, \ldots, x_{n}\right)=M_{f} \ltimes_{i}^{n} x_{i},
$$

where $M_{f} \in \mathscr{L}_{s \times k}$ is called the structural matrix of $F, y \in \Delta_{s}$, $s=s_{1} s_{2} \cdots s_{m}$, and $k=k_{1} k_{2} \cdots k_{n}$.

Lemma 6. Consider (5). For the sake of compactness, we denote $M_{f} W_{\left[k_{i}, \prod_{p=1}^{i-1} k_{p}\right]}=M$. For any $1 \leq i \leq n$, we split $M$ into $k_{i}$ equal-size blocks as $\left[B l k_{1}(M), \ldots, B l k_{k_{i}}(M)\right]$. If all the blocks are the same, then $x_{i}$ is a redundant variable. Thus, $y$ can be replaced by

$$
y=M_{f}^{\prime} x_{1} \cdots x_{i-1} x_{i+1} \cdots x_{n}
$$

where $M_{f}^{\prime}=B l k_{1}(M)=M_{f} W_{\left[k_{i}, \prod_{p=1}^{i-1} k_{p}\right]} \delta_{k_{i}}^{1}$

\section{Multivariable FLC Based on STP for Mobile Robot OSL}

Consider the linguistic control rules of the multivariable fuzzy system:

$$
\begin{aligned}
& R^{l}: \text { IF } x_{1} \text { is } A_{1}^{l}, \ldots, \text { and } x_{n} \text { is } A_{n}^{l} \text {, } \\
& \text { THEN } y_{1} \text { is } B_{1}^{l}, \ldots \text {, and } y_{m} \text { is } B_{m}^{l} \text {, }
\end{aligned}
$$

where $x_{i}$ and $y_{j}$ are linguistic variables representing the process state and the control variable, respectively. $R^{l}$ denotes the $l$ th fuzzy inference rule, where $l \in\{1, \ldots, L\}$, and $L$ is the number of fuzzy rules. $A_{i}, i=1, \ldots, n$, and $B_{j}, j=1, \ldots, m$, are the normalized fuzzy set of linguistic values on universes of discourses $X_{i}$ and $Y_{j}$, respectively. The control system is shown in Figure 1.

3.1. Controller Design of MFS with Complete Fuzzy Control Rules. The fuzzy control rules are in accordance with consistency and correctness. For the $n$ inputs and $m$ outputs fuzzy 
controller (7), let the number of the linguistic values of $x_{i}$ and $y_{j}$ be $k_{i}$ and $s_{j}$, respectively; that is,

$$
\begin{aligned}
& x_{i} \in \mathscr{D}_{k_{i}}, \\
& A_{i}=\left\{A_{i}^{1}, \ldots, A_{i}^{k_{i}}\right\}, \\
& y_{j} \in \mathscr{D}_{s_{j}}, \\
& B_{j}=\left\{B_{j}^{1}, \ldots, B_{j}^{s_{j}}\right\}, \ldots, n,
\end{aligned}
$$

We identify $A_{1}^{i_{1}} \sim \delta_{k_{1}}^{i_{1}} ; \ldots ; A_{n}^{i_{n}} \sim \delta_{k_{n}}^{i_{n}}, B_{1}^{j_{1}} \sim \delta_{s_{1}}^{j_{1}} ; \ldots ; B_{m}^{j_{m}} \sim$ $\delta_{s_{m}}^{j_{m}}, i_{1}=1, \ldots, k_{1} ; \ldots ; i_{n}=1, \ldots, k_{n} ; j_{1}=1, \ldots, s_{1} ; \ldots ; j_{m}=$ $1, \ldots, s_{m}$.

Then, (7) can be written as

$$
\begin{gathered}
R^{l} \text { : IF } x_{1}=\delta_{k_{1}}^{i_{1}}, \ldots, \text { and } x_{n}=\delta_{k_{n}}^{i_{n}}, \\
\text { THEN } y_{1}=\delta_{s_{1}}^{j_{1}}, \ldots, \text { and } y_{m}=\delta_{s_{m}}^{j_{m}} .
\end{gathered}
$$

Using the vector form of logical variables, we express the fuzzy controller as

$$
\begin{gathered}
y_{1}=M_{1} x, \\
y_{2}=M_{2} x, \\
\vdots \\
y_{m}=M_{m} x, \\
y=M_{f} x,
\end{gathered}
$$

where $y:=\ltimes_{j=1}^{m} y_{j}, x:=\ltimes_{i=1}^{n} x_{i}, M_{j} \in \mathscr{L}_{s_{j} \times k}, j=1, \ldots, m$, and $\operatorname{Col}_{i}\left(M_{f}\right)=\operatorname{Col}_{i}\left(M_{1}\right) \ltimes \cdots \ltimes \operatorname{Col}_{i}\left(M_{m}\right)$, where $\operatorname{Col}_{i}\left(M_{f}\right)$ denotes the $i$ th column of matrix $M_{f}$. For rules $l$ and $y_{j}=$ $M_{j} x$, since $x=\ltimes_{i=1}^{n} x_{i}=\delta_{k_{1}}^{i_{1}} \ltimes \cdots \ltimes \delta_{k_{n}}^{i_{n}}=\delta_{k}^{i}, y_{j}=\delta_{s_{j}}^{j_{j}}$, we have $\operatorname{Col}_{i}\left(M_{j}\right)=\delta_{s_{j}}^{j_{j}}$. If the fuzzy rules are complete, all the columns of $M_{j}, j=1, \ldots, m$ can be obtained. Then, we have the following result.

Theorem 7. The structural matrices $M_{j}, j=1, \ldots, m$ and $M_{f}$ of the fuzzy controller can be uniquely determined, if and only if the fuzzy rules of the fuzzy controller are complete.

Proof. Consider the following.

Sufficiency. For the fuzzy rules (9), let $x=x_{1} \ltimes \cdots \ltimes x_{n}$. Assume the fuzzy rules of the fuzzy controller are complete; that is, there are $k$ fuzzy rules. For the $l$ th, $l=1, \ldots, k$, fuzzy rule, we have $x=\delta_{k}^{i}$ and $y_{1}=\delta_{s_{1}}^{j_{1}}$. Then the $i$ th column of $M_{1}$ can be obtained as

$$
\operatorname{Col}_{i}\left(M_{1}\right)=\delta_{s_{1}}^{j_{1}}
$$

Repeating this procedure, one can obtain all the columns of $M_{1}$ if the fuzzy rules are complete. Similarly, all $M_{2}, \ldots, M_{m}$ and $M_{f}$ can be determined.

Necessity. If the structural matrices $M_{j}$ and $M_{f}$ of the fuzzy controller are uniquely determined, then all the columns of $M_{j}$ and $M_{f}$ are uniquely determined. Because one column of $M_{f}$ can generate one fuzzy rule, one can obtain $k$ fuzzy rules from $k$ columns of $M_{j}$ or $M_{f}$; that is, the fuzzy rules are complete.

Remark 8. If the rules are not complete, some columns of $M_{j}$ and $M_{f}$ can be determined. In this case, the model is not unique. In addition, uncertain columns of $M_{j}$ and $M_{f}$ can be chosen arbitrarily.

\subsection{Controller Design of MFS with Incomplete Fuzzy Control} Rules. The fuzzy control rules are also in accordance with consistency and correctness. We first define a kind of incidence matrix to express the dynamic connection of the inputs and the outputs for a fuzzy controller.

Definition 9. Consider a fuzzy controller with $m$ controls and $n$ input variables. An $m \times n$ matrix, $\mathscr{J}=\left(r_{j, i}\right) \in \mathscr{R}^{m \times n}$, is called its incidence matrix, if

$$
r_{j, i}= \begin{cases}1, & y_{j} \text { depends on } x_{i} \\ 0, & \text { otherwise. }\end{cases}
$$

Consider fuzzy controllers (9) and (10); the indegree $d\left(y_{j}\right)$ is the number of the inputs and it influences $y_{j}$ directly. From the incidence matrix of the fuzzy controller, we have

$$
d\left(y_{j}\right)=\sum_{k=1}^{n} r_{j k}, \quad j=1, \ldots, m .
$$

A set of controls (10) is said to be a feasible one to (9), if (9) satisfies (10). A feasible set of controls (10) with the indegree $d^{*}\left(y_{j}\right), j=1, \ldots, m$, is called a least indegree feasible set, if for any other realization with indegree $d\left(y_{j}\right), j=1, \ldots, m$, we have

$$
d^{*}\left(y_{j}\right) \leq d\left(y_{j}\right), \quad j=1, \ldots, m \text {. }
$$

We can use Lemma 6 to remove redundant variables and obtain a least indegree feasible set when the fuzzy rules are complete.

Assume a set of incomplete rules as

$$
\begin{aligned}
& R^{l} \text { : IF } x_{1} \text { is } A_{1}^{l}, \ldots \text {, and } x_{n} \text { is } A_{n}^{l}, \\
& \text { THEN } y_{1} \text { is } B_{1}^{l}, \ldots \text {, and } y_{m} \text { is } B_{m}^{l}, \\
& \qquad l \in\{1, \ldots, t\},
\end{aligned}
$$


where $R^{l}$ denotes the $l$ th fuzzy control rule, $t$ is the number of the control rules, and $t<k$.

Consider the controls $y_{j}=M_{j} x$. Using this set of fuzzy rules, some columns of the structural matrix $M_{j}$ can be determined. For instance

$$
\begin{aligned}
& M_{j} \\
& =\delta_{s_{j}}\left[\star \cdots \star c_{j_{1}} \star \cdots \star \cdots \star c_{j_{k}} \star \cdots \star\right.
\end{aligned}
$$

where " $\star$ " stands for the uncertain columns. Equation (17) is called the uncertain structural matrix. Let

$$
M_{j, i}:=M_{j} W_{\left[k_{i}, \prod_{p=1}^{i-1} k_{p}\right]}, \quad i=1, \ldots, n
$$

Then split it into $k_{i}$ equal blocks as

$$
M_{j, i}:=\left[\begin{array}{llll}
M_{j, i}^{1} & M_{j, i}^{2} & \cdots & M_{j, i}^{k_{i}}
\end{array}\right] .
$$

According to Lemma 6, we have the following result.

Proposition 10. The fuzzy control $y_{j}$ has an algebraic form which is independent of $x_{i}$, if and only if

$$
M_{j, i}^{1}=M_{j, i}^{2}=\cdots=M_{j, i}^{k_{i}}
$$

has a solution for uncertain elements.
Proof. Consider the following.

Sufficiency. Assume that (20) holds. By Lemma 5, the fuzzy control $y_{j}$ has an algebraic form which is independent of $x_{i}$.

Necessity. Assume the fuzzy control $y_{j}$ is independent of $x_{i}$; then $y_{j}$ remains invariant whenever $x_{i}=\delta_{k_{i}}^{q}, q=1, \ldots, k_{i}$. Thus

$$
M_{j} W_{\left[k_{i}, \prod_{p=1}^{i-1} k_{p}\right]} \delta_{k_{i}}^{1}=\cdots=M_{j} W_{\left[k_{i}, \prod_{p=1}^{i-1} k_{p}\right]} \delta_{k_{i}}^{k_{i}},
$$

which implies that (20) holds. The proof is completed.

Example 11. Consider a fuzzy controller, which has 4 inputs, $x_{1}, x_{3} \in \mathscr{D}_{2}, x_{2}, x_{4} \in \mathscr{D}_{3}$, and 2 outputs (controls), $y_{1} \in \mathscr{D}_{3}$ and $y_{2} \in \mathscr{D}_{4}$.

In the vector form, we assume that there are a set of control rules as follows:

$$
\begin{aligned}
& \text { IF } x_{1}=\delta_{2}^{1}, x_{2}=\delta_{3}^{1}, x_{3}=\delta_{3}^{1} \text { and } x_{4}=\delta_{2}^{1} \text {, THEN } \\
& y_{1}=\delta_{3}^{2} \text { and } y_{2}=\delta_{4}^{2} . \\
& \text { IF } x_{1}=\delta_{2}^{1}, x_{2}=\delta_{3}^{2}, x_{3}=\delta_{2}^{2} \text { and } x_{4}=\delta_{3}^{1} \text {, THEN } \\
& y_{1}=\delta_{3}^{2} \text { and } y_{2}=\delta_{4}^{4} . \\
& \text { IF } x_{1}=\delta_{2}^{1}, x_{2}=\delta_{3}^{3}, x_{3}=\delta_{2}^{2} \text { and } x_{4}=\delta_{3}^{3} \text {, THEN } \\
& y_{1}=\delta_{3}^{1} \text { and } y_{2}=\delta_{4}^{1} . \\
& \text { IF } x_{1}=\delta_{2}^{2}, x_{2}=\delta_{3}^{1}, x_{3}=\delta_{2}^{1} \text { and } x_{4}=\delta_{3}^{1} \text {, THEN } \\
& y_{1}=\delta_{3}^{1} \text { and } y_{2}=\delta_{4}^{3} . \\
& \text { IF } x_{1}=\delta_{2}^{2}, x_{2}=\delta_{3}^{2}, x_{3}=\delta_{2}^{1} \text { and } x_{4}=\delta_{3}^{3} \text {, THEN } \\
& y_{1}=\delta_{3}^{3} \text { and } y_{2}=\delta_{4}^{2} . \\
& \text { IF } x_{1}=\delta_{3}^{2}, x_{2}=\delta_{3}^{3}, x_{3}=\delta_{2}^{2} \text { and } x_{4}=\delta_{3}^{2} \text {, THEN } \\
& y_{1}=\delta_{3}^{3} \text { and } y_{2}=\delta_{4}^{4} .
\end{aligned}
$$

Now, we would like to get a least indegree feasible set of controls. Some columns of $M_{1}$ and $M_{2}$ can be identified as

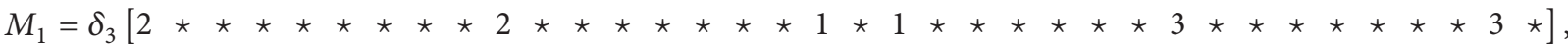

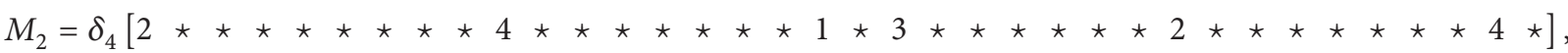

where " $\star$ " denotes the unknown element. Now, we check whether $x_{1}$ can be a redundant variable of $y_{1}$. Split $M_{1}$ into two equal blocks as $M_{1}=\left[\begin{array}{ll}M_{1}^{1} & M_{1}^{2}\end{array}\right]$, and let $M_{1}^{1}=M_{1}^{2}$ which yields the solution as

$$
\begin{aligned}
& M_{1}^{1}=M_{1}^{2}
\end{aligned}
$$

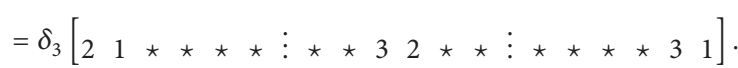

Thus, the control can be simplified as $y_{1}=M_{1}^{1} x_{2} x_{3} x_{4}$. Now, we check $x_{2}$. Splitting $M_{1}^{1}$ into three equal blocks as $\left[\begin{array}{lll}M_{1}^{11} & M_{1}^{12} & M_{1}^{13}\end{array}\right]$ and letting $M_{1}^{11}=M_{1}^{12}=M_{1}^{13}$, it can be updated as

$$
M_{1}^{11}=M_{1}^{12}=M_{1}^{13}=\delta_{3}\left[\begin{array}{llllll}
2 & 1 & 3 & 2 & 3 & 1
\end{array}\right],
$$

which satisfies $y_{1}=M_{1}^{11} x_{3} x_{4}$. Next, check $x_{3}$ and $x_{4}$. Since $M_{1}^{11} W_{[2,3]}=\delta_{3}\left[\begin{array}{llllll}2 & 2 & 1 & 3 & 3 & 1\end{array}\right], x_{3}$ and $x_{4}$ are not fabricated variables. Finally, we obtain $y_{1}=$ $\delta_{3}\left[\begin{array}{llllll}2 & 2 & 1 & 3 & 3 & 1\end{array}\right] x_{3} x_{4}$. Similarly, we have $y_{2}=$ $\delta_{4}\left[\begin{array}{llllll}2 & 4 & 3 & 4 & 2 & 1\end{array}\right] x_{3} x_{4}$.

And then, the least indegree realization can finally be obtained as

$$
\begin{aligned}
& y_{1}=\delta_{3}\left[\begin{array}{llllll}
2 & 2 & 1 & 3 & 3 & 1
\end{array}\right] x_{3} x_{4}, \\
& y_{2}=\delta_{4}\left[\begin{array}{llllll}
2 & 4 & 3 & 4 & 2 & 1
\end{array}\right] x_{3} x_{4} .
\end{aligned}
$$

3.3. Controller Design of MFS for Mobile Robot OSL. A great deal of sensor information needs to be processed rapidly for a mobile robot during the real-time searching process, 


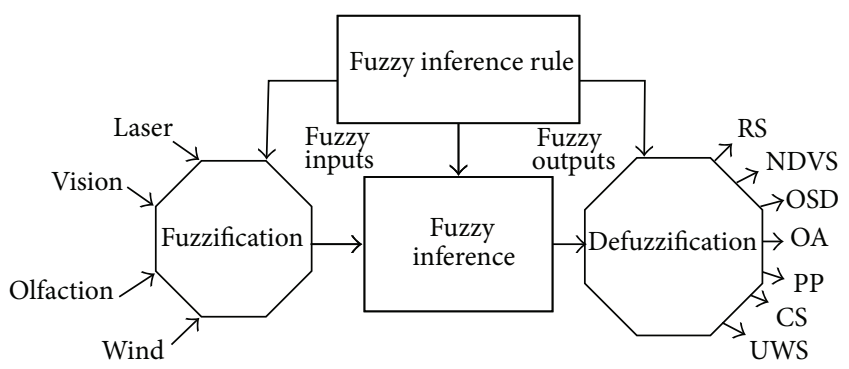

FIGURE 2: Fuzzy control based system for mobile robot OSL.

such as gas sensor (olfaction), camera (vision), wind sensor (wind speed and direction), laser sensor (distance), and electronic compass (position of robot). The mobile robot needs to make correct determination when different sensor information is required. In this paper, a MIMO fuzzy control based localization framework (shown in Figure 2) is set up in order to make full use of the diversity and complementary of multisensor information and obtain more detailed and accurate decision. The inputs are the multisensor information or the computed results of the sensor information. Here, the laser sensor information (LSI) is represented by the linguistic terms "near" and "far," vision information (VI) is "true" and "false," olfaction information (OI) is "too low," "normal," and "too high," and wind information (WI) is "true" and "false." And the outputs are several behaviors. In this paper, six behaviors are set up, including obstacle avoiding (OA), odor source declaration (OSD), nearest distance-based visual searching (NDVS), up-wind searching (UWS), path planning (PP), chemotaxis searching (CS), and random searching (RS).

We identify the following:

$$
\begin{aligned}
& \text { Inputs: LSI } \sim x_{1}, \mathrm{OI} \sim x_{2}, \mathrm{VI} \sim x_{3}, \mathrm{WI} \sim x_{4} . \\
& \text { Outputs: } \mathrm{OA} \sim \delta_{7}^{1}, \mathrm{GSD} \sim \delta_{7}^{2}, \mathrm{NDVS} \sim \delta_{7}^{3}, \mathrm{UWS} \sim \\
& \delta_{7}^{4}, \mathrm{PP} \sim \delta_{7}^{5}, \mathrm{CS} \sim \delta_{7}^{6}, \mathrm{RS} \sim \delta_{7}^{7}: \\
& \text { near } \sim \delta_{2}^{1}, \\
& \text { far } \sim \delta_{2}^{2},
\end{aligned}
$$

$$
\begin{array}{r}
\text { true } \sim \delta_{2}^{1}, \\
\text { false } \sim \delta_{2}^{2}, \\
\text { too low } \sim \delta_{3}^{1}, \\
\text { normal } \sim \delta_{3}^{2}, \\
\text { too high } \sim \delta_{3}^{3} .
\end{array}
$$
form.

Then, the fuzzy rules can be expressed into the following

IF $x_{1}=\delta_{2}^{1}, x_{2}=\delta_{3}^{1}, x_{3}=\delta_{2}^{1}$, and $x_{4}=\delta_{2}^{1}$, THEN $y=\delta_{7}^{1}$; IF $x_{1}=\delta_{2}^{1}, x_{2}=\delta_{3}^{1}, x_{3}=\delta_{2}^{1}$, and $x_{4}=\delta_{2}^{2}$, THEN $y=\delta_{7}^{1}$; IF $x_{1}=\delta_{2}^{1}, x_{2}=\delta_{3}^{1}, x_{3}=\delta_{2}^{2}$, and $x_{4}=\delta_{2}^{1}$, THEN $y=\delta_{7}^{1}$; IF $x_{1}=\delta_{2}^{1}, x_{2}=\delta_{3}^{1}, x_{3}=\delta_{2}^{2}$, and $x_{4}=\delta_{2}^{2}$, THEN $y=\delta_{7}^{1}$; IF $x_{1}=\delta_{2}^{1}, x_{2}=\delta_{3}^{2}, x_{3}=\delta_{2}^{1}$, and $x_{4}=\delta_{2}^{1}$, THEN $y=\delta_{7}^{1}$; IF $x_{1}=\delta_{2}^{1}, x_{2}=\delta_{3}^{2}, x_{3}=\delta_{2}^{1}$, and $x_{4}=\delta_{2}^{2}$, THEN $y=\delta_{7}^{1}$; IF $x_{1}=\delta_{2}^{1}, x_{2}=\delta_{3}^{2}, x_{3}=\delta_{2}^{2}$, and $x_{4}=\delta_{2}^{2}$, THEN $y=\delta_{7}^{1}$; IF $x_{1}=\delta_{2}^{1}, x_{2}=\delta_{3}^{2}, x_{3}=\delta_{2}^{2}$, and $x_{4}=\delta_{2}^{1}$, THEN $y=\delta_{7}^{1}$; IF $x_{1}=\delta_{2}^{1}, x_{2}=\delta_{3}^{3}, x_{3}=\delta_{2}^{1}$, and $x_{4}=\delta_{2}^{1}$, THEN $y=\delta_{7}^{2}$; IF $x_{1}=\delta_{2}^{1}, x_{2}=\delta_{3}^{3}, x_{3}=\delta_{2}^{1}$, and $x_{4}=\delta_{2}^{2}$, THEN $y=\delta_{7}^{2}$; IF $x_{1}=\delta_{2}^{1}, x_{2}=\delta_{3}^{3}, x_{3}=\delta_{2}^{2}$, and $x_{4}=\delta_{2}^{1}$, THEN $y=\delta_{7}^{1}$; IF $x_{1}=\delta_{2}^{1}, x_{2}=\delta_{3}^{3}, x_{3}=\delta_{2}^{2}$, and $x_{4}=\delta_{2}^{2}$, THEN $y=\delta_{7}^{1}$; IF $x_{1}=\delta_{2}^{2}, x_{2}=\delta_{3}^{1}, x_{3}=\delta_{2}^{1}$, and $x_{4}=\delta_{2}^{1}$, THEN $y=\delta_{7}^{3}$; IF $x_{1}=\delta_{2}^{2}, x_{2}=\delta_{3}^{1}, x_{3}=\delta_{2}^{1}$, and $x_{4}=\delta_{2}^{2}$, THEN $y=\delta_{7}^{3}$; IF $x_{1}=\delta_{2}^{2}, x_{2}=\delta_{3}^{1}, x_{3}=\delta_{2}^{2}$, and $x_{4}=\delta_{2}^{2}$, THEN $y=\delta_{7}^{7}$; IF $x_{1}=\delta_{2}^{2}, x_{2}=\delta_{3}^{1}, x_{3}=\delta_{2}^{2}$, and $x_{4}=\delta_{2}^{1}$, THEN $y=\delta_{7}^{7}$; IF $x_{1}=\delta_{2}^{2}, x_{2}=\delta_{3}^{2}, x_{3}=\delta_{2}^{1}$, and $x_{4}=\delta_{2}^{1}$, THEN $y=\delta_{7}^{5}$; IF $x_{1}=\delta_{2}^{2}, x_{2}=\delta_{3}^{2}, x_{3}=\delta_{2}^{1}$, and $x_{4}=\delta_{2}^{2}$, THEN $y=\delta_{7}^{5}$; IF $x_{1}=\delta_{2}^{2}, x_{2}=\delta_{3}^{2}, x_{3}=\delta_{2}^{2}$, and $x_{4}=\delta_{2}^{1}$, THEN $y=\delta_{7}^{4}$; IF $x_{1}=\delta_{2}^{2}, x_{2}=\delta_{3}^{2}, x_{3}=\delta_{2}^{2}$, and $x_{4}=\delta_{2}^{2}$, THEN $y=\delta_{7}^{6}$; IF $x_{1}=\delta_{2}^{2}, x_{2}=\delta_{3}^{3}, x_{3}=\delta_{2}^{1}$, and $x_{4}=\delta_{2}^{1}$, THEN $y=\delta_{7}^{5}$; IF $x_{1}=\delta_{2}^{2}, x_{2}=\delta_{3}^{3}, x_{3}=\delta_{2}^{1}$, and $x_{4}=\delta_{2}^{2}$, THEN $y=\delta_{7}^{5}$; IF $x_{1}=\delta_{2}^{2}, x_{2}=\delta_{3}^{3}, x_{3}=\delta_{2}^{2}$, and $x_{4}=\delta_{2}^{1}$, THEN $y=\delta_{7}^{4}$; IF $x_{1}=\delta_{2}^{2}, x_{2}=\delta_{3}^{3}, x_{3}=\delta_{2}^{2}$, and $x_{4}=\delta_{2}^{2}$, THEN $y=\delta_{7}^{6}$; from the above form of the fuzzy rules, we can obtain the structure matrix:

$$
M_{f}=\delta_{7}\left[\begin{array}{llllllllllllllllllllllll}
1 & 1 & 1 & 1 & 1 & 1 & 1 & 1 & 2 & 2 & 1 & 1 & 3 & 3 & 7 & 7 & 5 & 5 & 4 & 6 & 5 & 5 & 4 & 6
\end{array}\right] ;
$$

then $y=M_{f} x_{1} x_{2} x_{3} x_{4}$.

$$
\begin{aligned}
M_{f} & =\delta_{7}\left[\begin{array}{lllllllllllllllllllllllll}
1 & 1 & 1 & 1 & 1 & 1 & 1 & 1 & 2 & 2 & 1 & 1 & \vdots & 3 & 3 & 7 & 7 & 5 & 5 & 4 & 6 & 5 & 5 & 4 & 6
\end{array}\right], \\
M_{f} W_{[3,2]} & =\delta_{7}\left[\begin{array}{llllllllllllllllllllllll}
1 & 1 & 1 & 1 & 3 & 3 & 7 & 7 & 1 & 1 & 1 & 1 & 5 & 5 & 4 & 6 & 2 & 2 & 1 & 1 & 5 & 5 & 4 & 6
\end{array}\right], \\
M_{f} W_{[2,6]} & =\delta_{7}\left[\begin{array}{llllllllllllllllllllllll}
1 & 1 & 1 & 1 & 2 & 2 & 3 & 3 & 5 & 5 & 5 & 5 & 1 & 1 & 1 & 1 & 1 & 1 & 7 & 7 & 4 & 6 & 4 & 6
\end{array}\right], \\
M_{f} W_{[2,12]} & =\delta_{7}\left[\begin{array}{llllllllllllllllllllllll}
1 & 1 & 1 & 1 & 2 & 1 & 3 & 7 & 5 & 4 & 5 & 4 & 1 & 1 & 1 & 1 & 2 & 1 & 3 & 7 & 5 & 6 & 5 & 6
\end{array}\right] .
\end{aligned}
$$




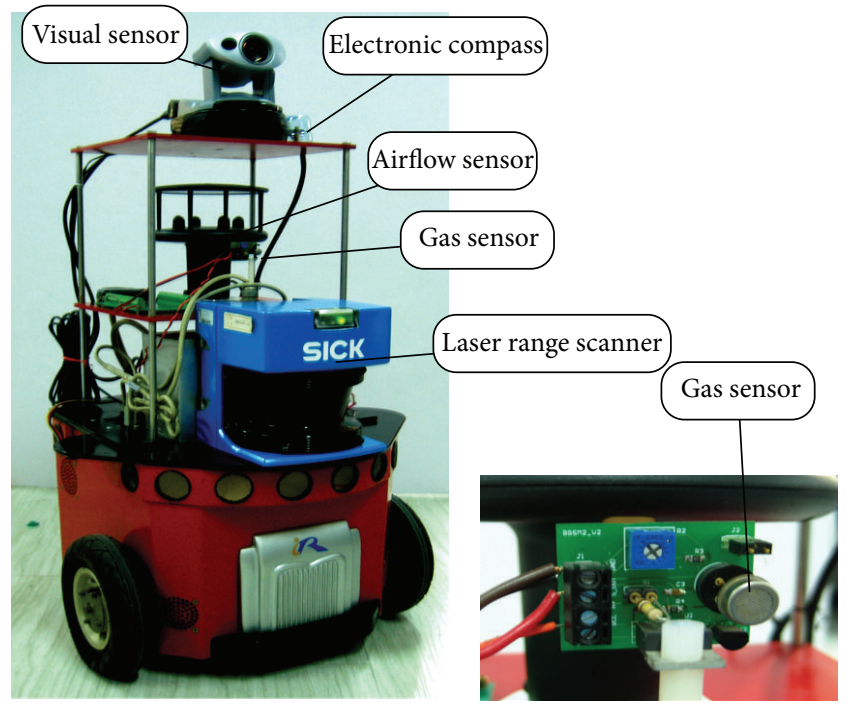

Figure 3: Experimental platform of mobile robot OSL.

Obviously, we know $x_{1}, x_{2}, x_{3}$, and $x_{4}$ are not redundant variables of $y$.

Assume that LSI is "near," OI is "too high," VI is "true," and WI is "true" or "false"; we have $y=M_{f} x_{1} x_{2} x_{3} x_{4}=$ $M_{f} \delta_{2}^{1} \delta_{3}^{3} \delta_{2}^{1} \delta_{2}^{1}=\delta_{7}^{2}$ or $y=M_{f} x_{1} x_{2} x_{3} x_{4}=M_{f} \delta_{2}^{1} \delta_{3}^{3} \delta_{2}^{1} \delta_{2}^{2}=\delta_{7}^{2}$, which means GSD.

\section{Experimental Results and Analysis}

The proposed method is verified using real robot experiments. The mobile robot platform and the odor source are shown in Figure 3. A PTZ camera (EVI-D100P, Sony), a gas sensor (MiCS-5135, e2v Technologies (UK) Ltd.), an anemometer (WindSonic, Gill), a laser rangefinder (LMS200, Sick AG), and an electronic compass were mounded on the robot. The PTZ is 1.3 meters high from the ground. The size of each sampled image is 320240 pixels. The computer (CPU: 3.0 GHz, RAM: 1.0 GBytes) is used in this paper.

4.1. The Experimental Result with No Vision and Olfaction. The mobile robot searches the whole scene to find the odor plume using random searching (RS) methods when there is no vision and olfaction information.

LSI is "far," OI is "too low," VI is "false," and WI is "true" or "false"; we have

$$
\begin{array}{r}
y=M_{f} x_{1} x_{2} x_{3} x_{4}=M_{f} \delta_{2}^{2} \delta_{3}^{1} \delta_{2}^{2} \delta_{2}^{1}=\delta_{7}^{7}, \\
\text { or } y=M_{f} x_{1} x_{2} x_{3} x_{4}=M_{f} \delta_{2}^{2} \delta_{3}^{1} \delta_{2}^{2} \delta_{2}^{2}=\delta_{7}^{7},
\end{array}
$$

which means RS. Figure 4 shows the searching trajectory. The robot starts spiral surge with a certain radius (the radius is $377 \mathrm{~mm}$ in this paper) from the initial position (the black solid dot in Figure 4). The blue dots is the moving trajectory.

4.2. The Experimental Result with Vision. Traditionally, random searching methods are used for plume finding when

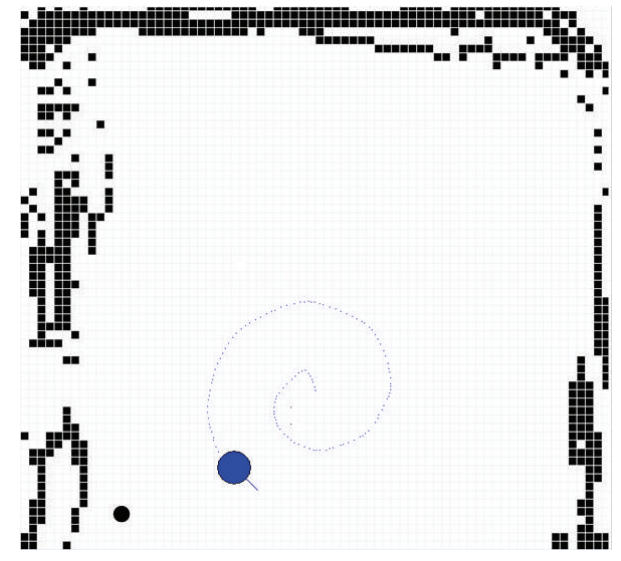

FIGURE 4: Random searching trajectory of mobile robot OSL.

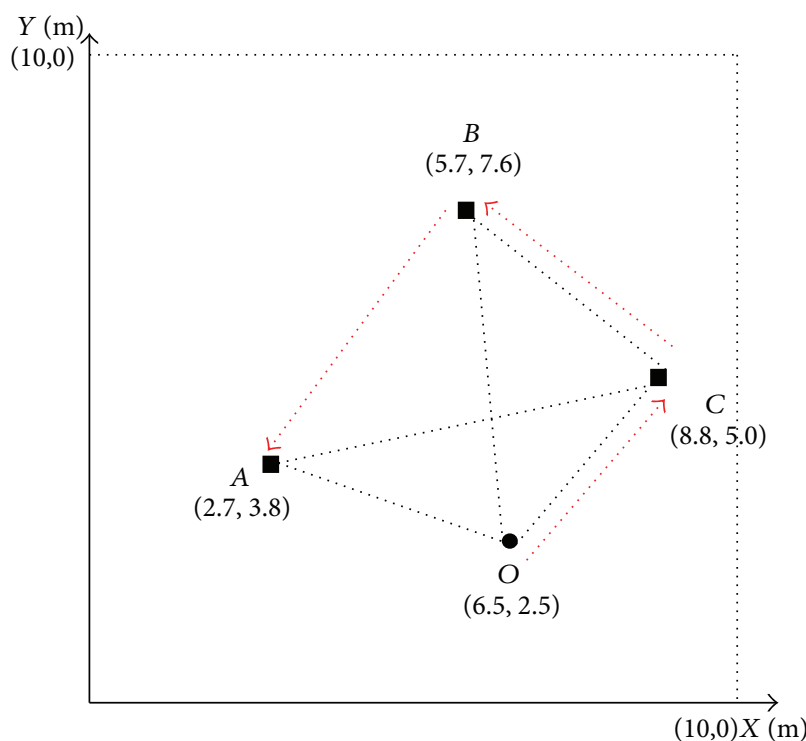

FIGURE 5: NDVS strategy of mobile robot OSL.

there is no olfaction. However, these methods have the same hypothesis that the probabilities of the gas leakage source appearing in the scene are equal, which is obviously inconsistent with the actual situation. Because the probabilities of gas leakage source in some areas is big and others may be small, thus, these random searching methods have certain blindness. If some potential gas sources are determined using vision in advance and then drives the robot to check the relative plausible areas firstly, it would overcome the blindness of random searching efficiently in a certain degree.

LSI is "far," OI is "too low," VI is "true," and WI is "true" or "false"; we have

$$
\begin{array}{r}
y=M_{f} x_{1} x_{2} x_{3} x_{4}=M_{f} \delta_{2}^{2} \delta_{3}^{1} \delta_{2}^{1} \delta_{2}^{1}=\delta_{7}^{3}, \\
\text { or } y=M_{f} x_{1} x_{2} x_{3} x_{4}=M_{f} \delta_{2}^{2} \delta_{3}^{1} \delta_{2}^{1} \delta_{2}^{2}=\delta_{7}^{3},
\end{array}
$$

which means NDVS. The optimal strategy is shown in Figure 5. If only one plausible area is existent in the scene, the robot moves to the area directly to check. If more 

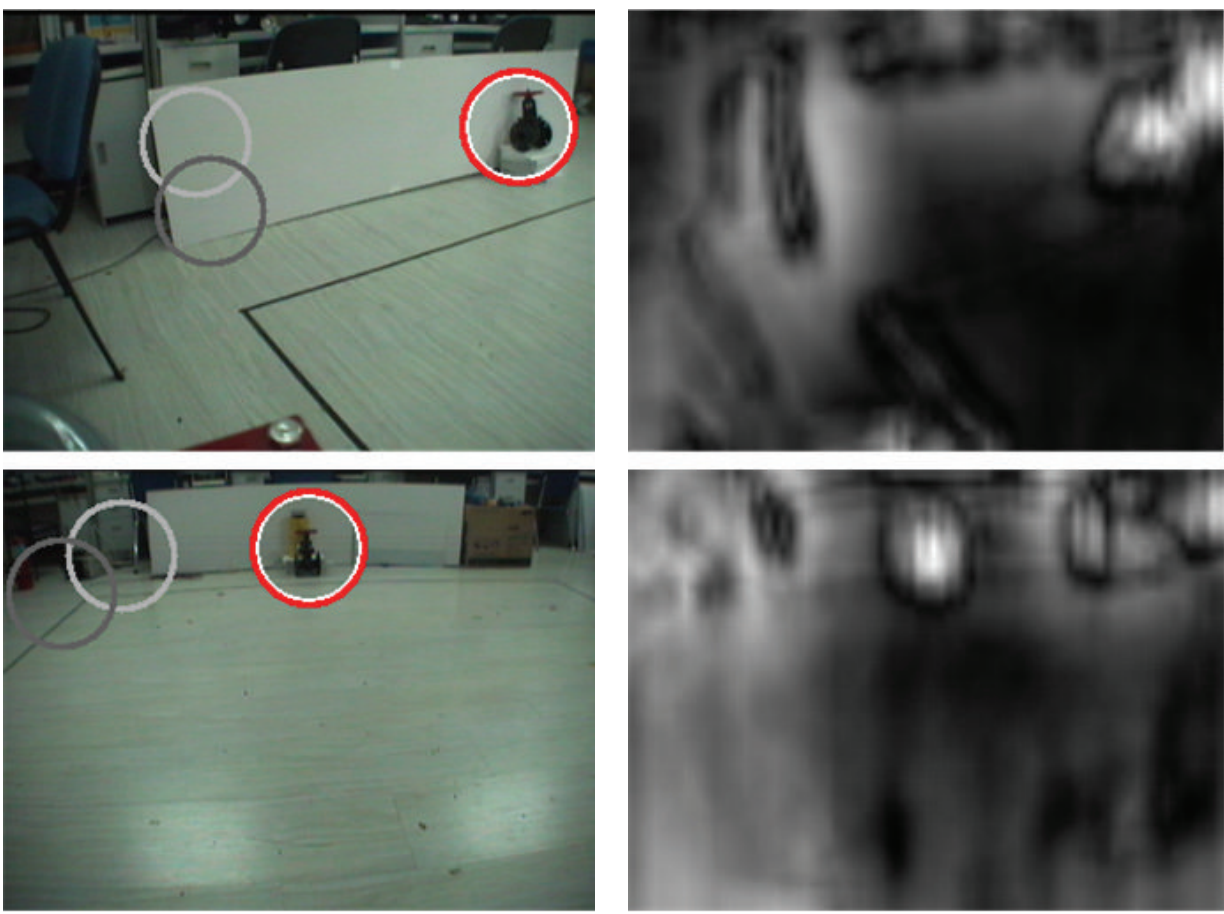

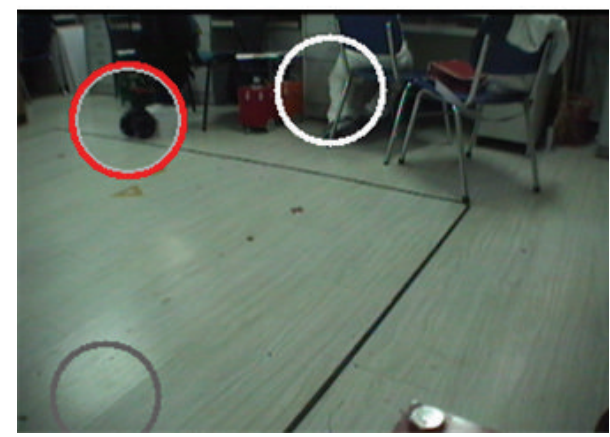

(a)

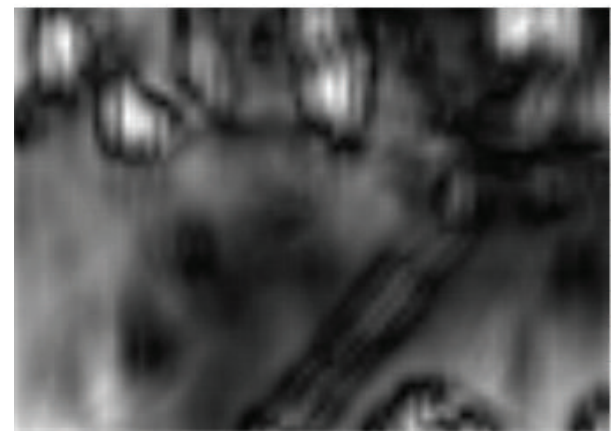

(b)

FIGURE 6: Scene images and the relative saliency maps.

plausible areas are existent it needs to plan the searching path to improve the searching efficiency. A recursive optimal searching strategy (NDVS: nearest distance based visual searching) is proposed in this paper because it cannot be determined in advance which one will find the gas source.

In Figure $4, O$ is the initial position $(6.5,2.5)$ and $A$ $(2.7,3.8), B(5.7,7.6)$, and $C(8.8,5.0)$ are the plausible areas obtained using top-down visual attention mechanism and shape analysis [13] to the vision information. The distances between the initial position and the plausible areas are $4.02 \mathrm{~m}$, $5.16 \mathrm{~m}$, and $3.40 \mathrm{~m}$, respectively. Thus, the robot moves to the nearest area (point $C$ ). If there is no gas source, the next target from $A$ and $B$ is selected according to the distance to $C$. $L_{C B}$ $(2.52 \mathrm{~m})$ is less than $L_{C A}(6.22 \mathrm{~m})$. Thus, $B$ is the next.

Figure 6(a) is the scene images in which the three white to gray circles represent the visual computing results (the most three saliency regions) and the red circle represents the potential gas source determined by using shape analysis. Figure 6(b) is the relative saliency map.
In Figure 7, point $O$ (red solid dot) is the initial position of the robot, $A, B$, and $C$ are the plausible areas, the big blue dot represents the robot, and the blue dots are the searching trajectory.

4.3. The Experimental Result with Vision and Olfaction. When vision, olfaction, and wind information are efficient the robot starts to make decision where to go, that is, path planning (PP). LSI is "far," OI is "normal," VI is "true," and WI is "true" or "false"; we have $y=M_{f} x_{1} x_{2} x_{3} x_{4}=$ $M_{f} \delta_{2}^{2} \delta_{3}^{2} \delta_{2}^{1} \delta_{2}^{1}=\delta_{7}^{5}$ or $y=M_{f} x_{1} x_{2} x_{3} x_{4}=M_{f} \delta_{2}^{2} \delta_{3}^{2} \delta_{2}^{1} \delta_{2}^{2}=\delta_{7}^{5}$ which means PP. The searching result is shown in Figure 8.

The red dot line is the trajectory of the mobile robot and the big red round is the start position. At the beginning there is no gas concentration, the robot moves toward point $A$ (points $A, B$, and $C$ are the plausible areas) by using NDVS method. And in the moving process gas concentration is detected; then the robot adjusts the moving direction constantly according to the gas concentration and wind information. When both laser information and vision are 


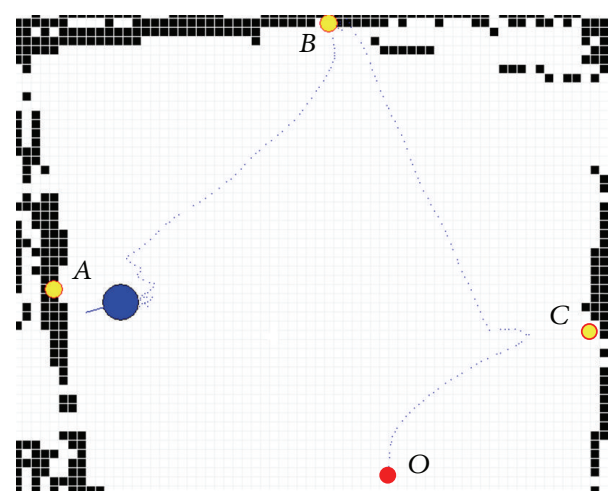

FIGURE 7: Real-time NDVS searching result.

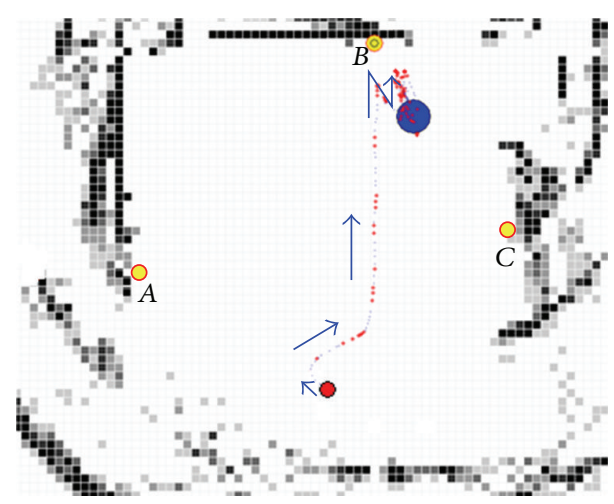

FIGURE 8: OSD trajectory of mobile robot OSL.

efficient and the gas concentration is detected constantly, the obstacle is declared as the gas source. LSI is "near," OI is "too high," VI is "true," and WI is "true" or "false"; we have $y=$ $M_{f} x_{1} x_{2} x_{3} x_{4}=M_{f} \delta_{2}^{1} \delta_{3}^{3} \delta_{2}^{1} \delta_{2}^{1}=\delta_{7}^{2}$ or $y=M_{f} x_{1} x_{2} x_{3} x_{4}=$ $M_{f} \delta_{2}^{1} \delta_{3}^{3} \delta_{2}^{1} \delta_{2}^{2}=\delta_{7}^{2}$, which means OSD.

In Figure 8, the point $B$ is real source. Once the avoiding behavior actives, that is, the laser information is efficient, it will drive the mobile robot move away from the obstacle. But the computing results with olfaction and vision drive the robot toward the area of the obstacle. Thus, the robot will keep wandering near the area and the potential gas source is declared as the real source ( $B$ is real source).

\section{Conclusion}

In this paper, the multivariable fuzzy logic controller based on semitensor product (STP) for mobile robot OSL is designed. Using the basic properties of STP, the complex fuzzy control rules, and fuzzy logic inference are converted into an algebraic form. The multisensor information is the inputs of the fuzzy control system and the relative searching strategies are the outputs. The proposed multivariable fuzzy control system can activate relative searching strategies according to the timely multisensor information detected by the mobile robot, which makes the robot generate an optimization strategy to deal with the dynamic, complex, and unstructured environments. Compared with the classic olfaction-based odor source localization methods, the presented method can overcome the blindness of plume finding to a certain degree; that is, the traditional algorithms for plume finding are random searching without odor information and the mobile robot will check the scene with equal probability. Actually, the probability of suspected odor source in the scene is different. Thus, it will help to find the plume with the aid of more sensors, such as cameral. Therefore, the proposed method can make up the blindness of the olfaction-based ones to a certain degree. Equally important, any searching strategy can be updated in this framework and has no influence on the others whether based on a single sensor information or multisensor information. The proposed localization framework can degenerate into the traditional olfaction-based localization system. Most importantly, we gave an in-depth study on mobile robot odor source localization from the angle of mathematics which can reach the theory of the mobile robot odor source localization. The reliability and robustness of the proposed method are validated with the real robot experiments.

\section{Conflict of Interests}

The authors declare that they have no competing interests.

\section{Acknowledgments}

The research work of this paper is sponsored by National Natural Science Foundation (61374065) and the Research Fund for the Taishan Scholar Project of Shandong Province of China.

\section{References}

[1] M. A. Frye, M. Tarsitano, and M. H. Dickinson, "Odor localization requires visual feedback during free flight in Drosophila melanogaster," Journal of Experimental Biology, vol. 206, no. 5, pp. 843-855, 2003.

[2] J. Z. Guo and A. K. Guo, "Crossmodal interactions between olfactory and visual learning in Drosophila," Science, vol. 309, no. 5732, pp. 307-310, 2006.

[3] A. Mafra-Neto and R. T. Carde, "Fine-scale structure of pheromone plumes modulates upwind orientation of flying moths," Nature, vol. 369, no. 6476, pp. 142-144, 1994.

[4] R. A. Russell, "Survey of robotic applications for odor-sensing technology," The International Journal of Robotics Research, vol. 20, no. 2, pp. 144-162, 2001.

[5] Q.-H. Meng and F. Li, "Review of active olfaction," Robot, vol. 28, no. 1, pp. 89-96, 2006.

[6] A. J. Lilienthal, A. Loutfi, and T. Duckett, "Airborne chemical sensing with mobile robots," Sensors, vol. 6, no. 11, pp. 1616-1678, 2006.

[7] W. Naeem, R. Sutton, and J. Chudley, "Chemical plume tracing and odour source localisation by autonomous vehicles," The Journal of Navigation, vol. 60, no. 2, pp. 173-190, 2007.

[8] G. Kowadlo and R. A. Russell, "Robot odor localization: a taxonomy and survey," The International Journal of Robotics Research, vol. 27, no. 8, pp. 869-894, 2008. 
[9] H. Ishida, Y. Wada, and H. Matsukura, "Chemical sensing in robotic applications: a review," IEEE Sensors Journal, vol. 12, no. 11, pp. 3163-3173, 2012.

[10] G. Kowadlo, D. Rawlinson, R. A. Russell, and R. Jarvis, "Bimodal search using complementary sensing (olfaction/vision) for odour source localisation," in Proceedings of the IEEE International Conference on Robotics and Automation (ICRA' 06), pp. 2041-2046, Piscataway, NJ , USA, May 2006.

[11] H. Ishida, H. Tanaka, H. Taniguchi, and T. Moriizumi, "Mobile robot navigation using vision and olfaction to search for a gas/odor source," Autonomous Robots, vol. 20, no. 3, pp. 231238, 2006.

[12] P. Jiang, M. Zeng, Q.-H. Meng, F. Li, and Y.-H. Li, "A novel object recognition method for mobile robot localizing a single odor/gas source in complex environments," in Proceedings of the IEEE International Conference on Robotics, Automation and Mechatronics (RAM '08), pp. 1-5, IEEE, Chengdu, China, September 2008.

[13] P. Jiang, Q.-H. Meng, and M. Zeng, "Mobile robot gas source localization via top-down visual attention mechanism and shape analysis," in Proceedings of the 8th World Congress on Intelligent Control and Automation (WCICA '10), pp. 1818-1823, Jinan, China, July 2010.

[14] P. Jiang and Y. Zhang, "Least square estimation based mobile robot gas source localization in stable-airflow environment," in Proceedings of the 31st Chinese Control Conference (CCC '12), pp. 7000-7004, IEEE, Hefei, China, July 2012.

[15] P. Jiang and Y. Zhang, "Subsumption architecture based mobile robot gas source localization in time-variant-airflow environment," in Proceedings of the 31st Chinese Control Conference (CCC '12), pp. 4814-4819, Hefei, China, July 2012.

[16] B. Khaleghi, A. Khamis, F. O. Karray, and S. N. Razavi, "Multisensor data fusion: a review of the state-of-the-art," Information Fusion, vol. 14, no. 1, pp. 28-44, 2013.

[17] K. W. Chen, Z. P. Zhang, and J. Long, "Multisource information fusion: issues, research progress and new trends," Computer Science, vol. 40, no. 8, pp. 6-13, 2013.

[18] G. Feng, "A survey on analysis and design of model based fuzzy control systems," IEEE Transactions on Fuzzy Systems, vol. 14, no. 5, pp. 676-697, 2006.

[19] G. Feng, Analysis and Synthesis of Fuzzy Control System: A Modelbased Approach, CRC Press, Boca Raton, Fla, USA, 2010.

[20] D. Z. Cheng, H. S. Qi, and Z. Q. Li, Analysis and Control of Boolean Networks: A Semi-Tensor Product Approach, Springer, London, UK, 2011.

[21] H. T. Li and Y. Z. Wang, "Boolean derivative calculation with application to fault detection of combinational circuits via the semi-tensor product method," Automatica, vol. 48, no. 4, pp. 688-693, 2012.

[22] D. Z. Cheng, H. S. Qi, and Z. Q. Li, "Model construction of Boolean network via observed data," IEEE Transactions on Neural Networks, vol. 22, no. 4, pp. 525-536, 2011.

[23] Y. Z. Wang, C. H. Zhang, and Z. B. Liu, "A matrix approach to graph maximum stable set and coloring problems with application to multi-agent systems," Automatica, vol. 48, no. 7 , pp. 1227-1236, 2012. 


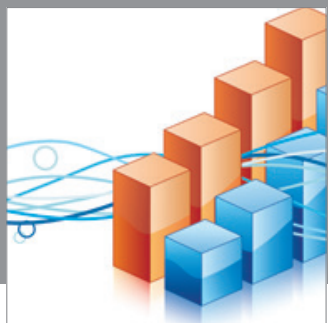

Advances in

Operations Research

mansans

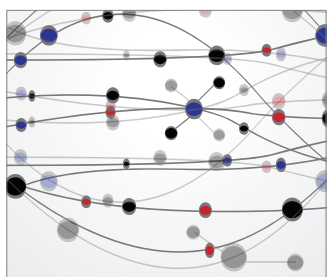

The Scientific World Journal
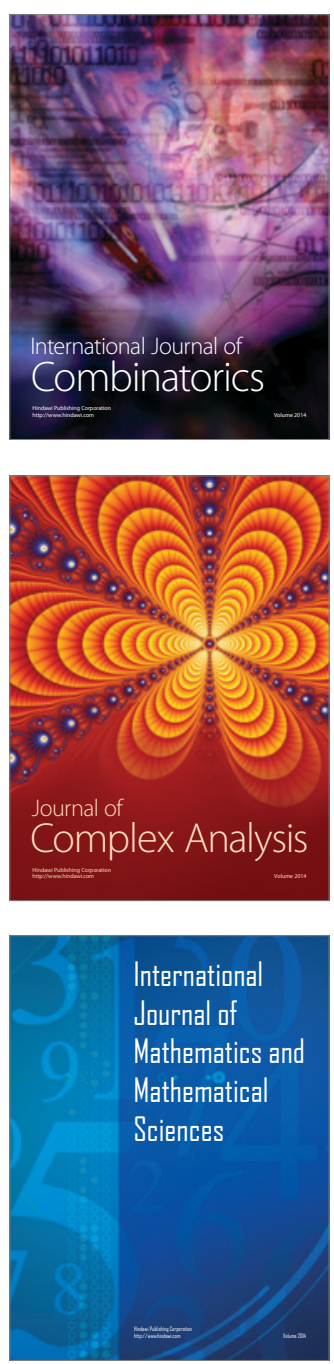
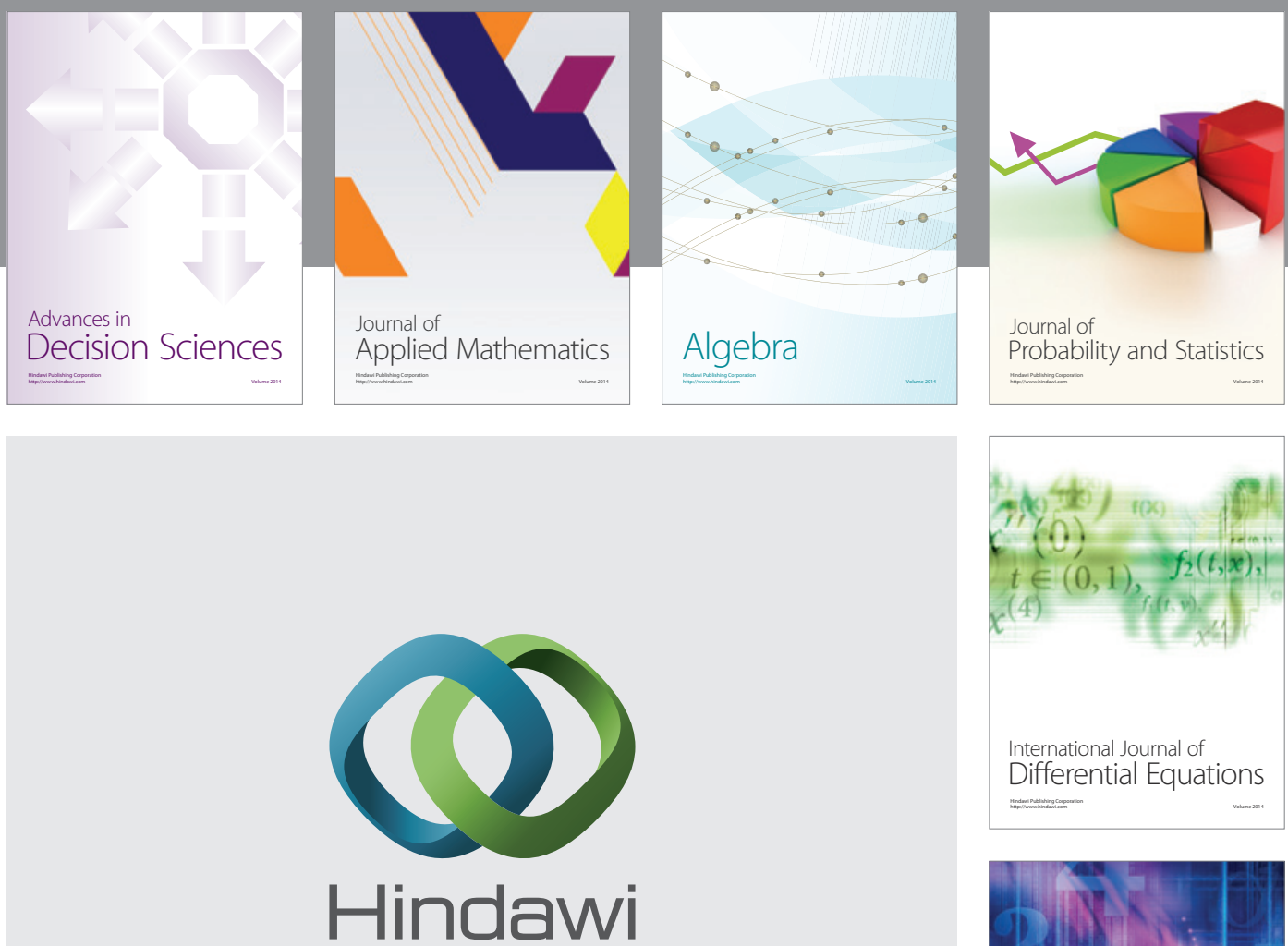

Submit your manuscripts at http://www.hindawi.com
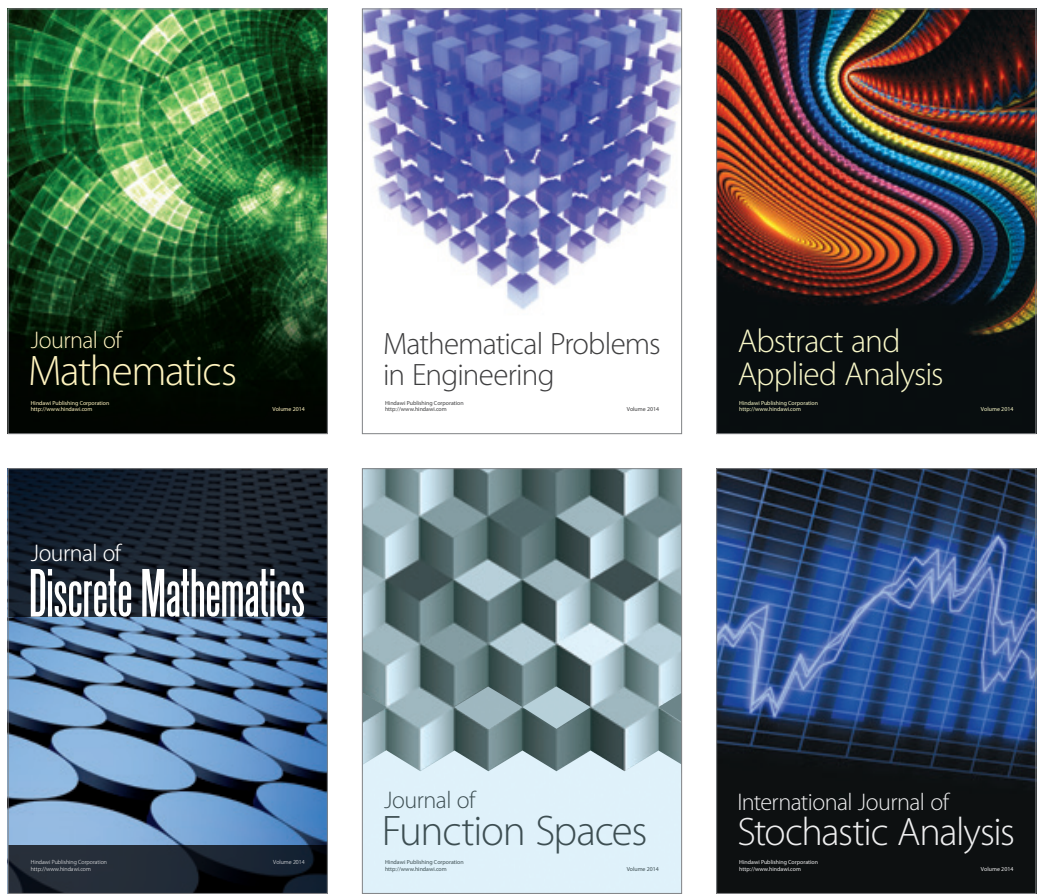

Journal of

Function Spaces

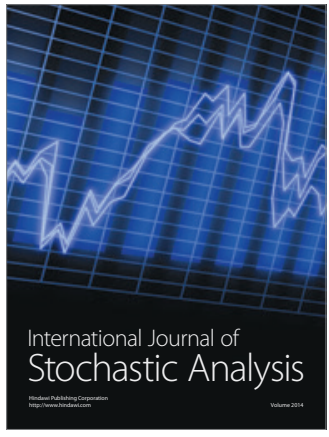

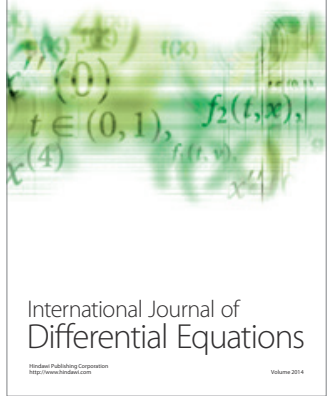
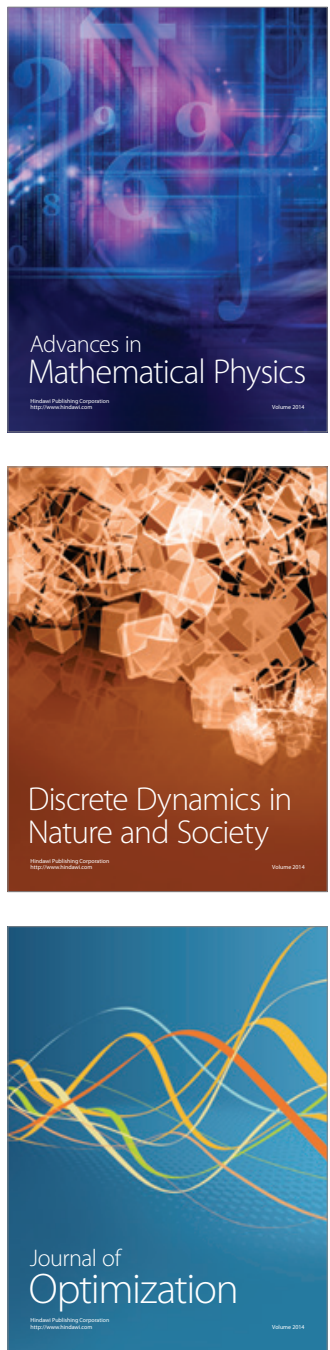